\title{
Tetralogy of Fallot with Anomalous Left Anterior Descending Coronary Artery :A Rare Repair - A Case Report and Review of Literature
}

\author{
MD. MAZIBUR RAHMAN ${ }^{1}$, SIBA PADA ROY ${ }^{2}$, JALAL UDDIN ${ }^{1}$ \\ ${ }^{1}$ Department of Cardiac surgery, Ibrahim Cardiac Hospital \& Research Institute, Shahbag, Dhaka, ${ }^{2}$ Department of \\ Cardiac Anaesthesiology, Ibrahim Cardiac Hospital \& Research Institute, Shahbag, Dhaka \\ Address of Correspondence: Dr. Md. Mazibur Rahman, Associate Professor and Consultant, Department of Cardiac surgery, \\ Ibrahim Cardiac Hospital \& Research Institute(ICHRI), 122, Kazi Nazrul Islam Avenue, Shahbag, Dhaka, \\ E-mail:drmuzib2009@yahoo.com
}

\begin{abstract}
:
Surgical total correction of Fallot's tetralogy (TOF) with coronary artery anomalies is associated with an increased risk for mortality and morbidity. We performed Trans-ventricular and Trans-annular autologous pericardial patchplasty with the incorporation of a monocusp valve at pulmonary annular level after excision of parietal and septal bands and anastomosis of left internal mammary artery (LIMA) at the distal segment of deliberately sacrificed left anterior descending coronary artery (LAD) in a 20-year young male patient in whom the LAD coronary artery arose from the right sinus of valsalva anomalously just next to the right coronary artery (RCA). The large ventricular septal defect (VSD) was closed with Dacron patch but the small atrial septal defect (ASD) was closed directly with suture. A large patent ductus arteriosus (PDA) and a major aortopulmonary collateral (MAPCA) were ligated before cardiopulmonary bypass (CPB). The patient was discharged without any problem.
\end{abstract}

Keywords: Tetralogy of Fallot, Left internal mamary artery, Left anterior descending coronary artery, Ventricular septal defect, Major aorto-pulmonary collateral artery and Right ventricular outflow tract obstruction.

\section{Introduction:}

Congenital coronary anomalies are a recognized cause of myocardial ischaemia and sudden cardiac death, especially among children and young adults. ${ }^{1}$ The prevalence of congenital coronary anomalies has been estimated at approximately $0.85 \%-1 \%$ of general population and the incidence of coronary artery anomalies is $2 \%$ to $8 \%$ in

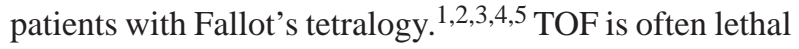
if untreated, it now has a good prognosis with timely surgical intervention. The best age for repair remains controversial but can be done at any age safely with low operative risk but should provide good relief of right ventricular outflow tract obstruction (RVOTO) to prevent progression of right ventricular (RV) hypertrophy. Proponents for primary neonatal repair cite factors such as prevention of time related end organ damage from cyanosis , removal of stimulus for RV hypertrophy and fibrosis, improved lung development (vascular and alveolar), avoidance of deleterious effects and risks of palliative shunts and psychosocial -economic issues. Surgical approach in patients having one coronary artery crossing the obstructed and hypertrophied right ventricular outflow tract (RVOT) is challenging and associated with increased operative mortality and morbidity. ${ }^{3}$ Presurgical documentation of coronary artery anomalies is essential for the most appropriate surgical approach. Echocardiographic assessment, coronary angiography, CT angiogram or recently developed electrocardiography-gated multidetector row CT (MDCT)a noninvasive, accurately imaging technique to visualize the coronary arteries from their origin, course and termination and is superior to conventional coronary angiogram. 6,7 Various techniques have been described to establish the continuity between the right ventricle and the pulmonary artery. The type of surgical reconstruction is individualized depending on both the anatomy of the RVOT and the course of the coronary artery over it. Techniques for RVOT reconstruction include an oblique ventriculotomy, tailored ventriculotomy, two patch repair, translocation of the main pulmonary artery, transannular repair below a mobilized coronary artery and pulmonary homograft conduit. ${ }^{8}$

We performed transventricular and transannular patchplasty with a monocusp after excision of septoparietal fibromuscular bands and anastomosis of LIMA to distal segment of sacrificed left anterior descending artery(LAD) in an adult patient. This corrective 
technique is appropriate in adult patients with anomalous non-tortuous coronaries which do not allow dissection and mobilization with the possibility of stress.

\section{Case Report:}

A 20-year-young man was admitted to our centre with symptoms of effort intolerence (NYHA class II). Precordial examination revealed grade 4/6 systolic ejection murmur heard along the left side of sternum and single S2. ECG showed right atrial (RA) enlargement with bi-ventricular hypertrophy. Chest roentgenogram demonstrated moderate cardiomegaly with boot shaped heart. Echocardiography revealed a large perimembranous ventricular septal defect(VSD), overriding of aorta $\sim 50 \%$, pulmonary valve annulu was hypoplastic having $13 \mathrm{~mm}$. diameter with bicuspid deformed leaflets forming a stenotic valve with a pressure gradient of $80 \mathrm{mmHg}$. Right pulmonary artery (RPA), left pulmonary artery (LPA) with short and narrow main pulmonary artery (MPA) were 9,10, and 12 $\mathrm{mm}$ in diameter respectively. CT aortopulmonary angiogram: the right coronary artery was arising from the right sinus of valsalva. The LAD coronary artery arose from the right aortic sinus of valsalva anomalously, just next to the right coronary artery (Fig.1). The LAD coronary artery passes anterior to the RVOT non-tortuously and descended along the anterior interventricular groove.

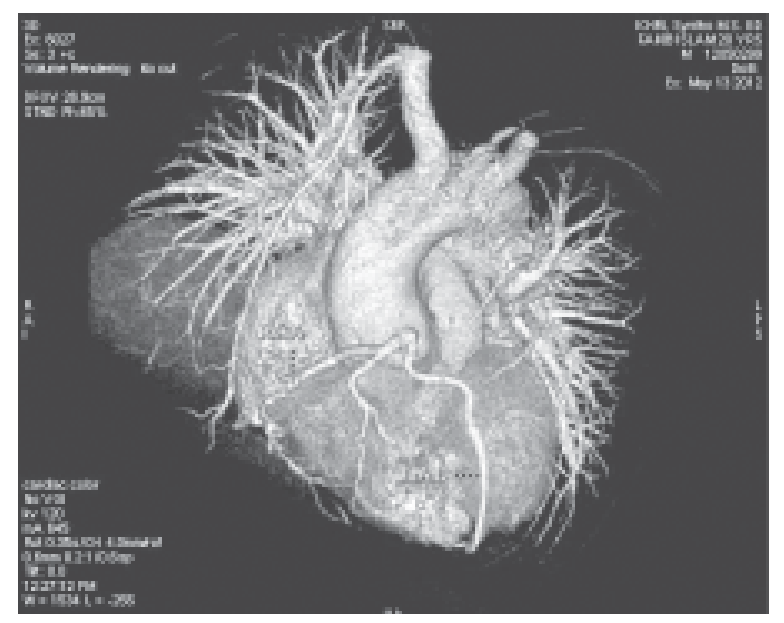

Fig.-1: CT aortopulmonary angiogram showing the left anterior descending (LAD) artery arose separately in the right aortic sinus of Valsalva together with the right coronary artery (RCA). The non-tortuous $L A D$ is crossing over the right ventricular outflow tract (RVOT).

\section{Surgical technique:}

After trans-sternal median thoracotomy, a pericardial patch was prepared and treated with $0.9 \%$ gluteraldehyde solution. Surgical exploration confirmed that both the LAD and the right coronary artery arose from the right coronary sinus separately. The LAD artery crossed across the RVOT and the RCA gave large perforating conal and RV branches(Fig. 1). The large PDA and a MAPCA were ligated. The LIMA was prepared without cutting the distal end. CPB was established with aortic and bicaval cannulation Myocardial preservation was achieved with antegrade cold blood cardioplegia repeated in every 20 minutes. Right atriotomy(RA-tomy) and vertical pulmonary arteriotomy (PA-tomy)was done. The bicuspid, domed and stenotic pulmonary valve (PV) on a hypoplastic annulus measuring $13 \mathrm{~mm}$. in diameter which was incompatible for a $47 \mathrm{~kg}$. man to pass a 18 size Hagar dilator. So PA-tomy was extended through annulus toward infundibulum for resection of septo-parietal fibromuscular bands. The LAD was in such a position over the RVOT, so that distal right ventriculotomy (RV-tomy) was adequately impossible without deliberately sacrifice of LAD which was just 1.2 $\mathrm{cm}$. below the pulmonary annulus. Moreover, the septoparaital bands were huge in amount which was difficult to remove completely through RA. A large VSD of $2.0 \mathrm{~cm}$ diameter was closed using a Dacron patch through a RVOT incision which provided better exposure than that made through the right atrium. The pulmonary pericardial annuloplasty having a monocusp valve which provided the pulmonary annulas $18 \mathrm{~mm}$. in diameter as well as wide infundibular passage. The LIMA was anastomosed directly with the distal segment of the divided LAD. The RA was closed after direct closure of ASD and the cross-clamp was removed.

Aortic, right ventricular and pulmonary artery pressures were 110/60 mmHg, 55/4 mmHg. and 20/7 mmHg. respectively at the end of CPB. His stay in ICU was uneventful with good haemodynamic. The patient was extubated on the same day of operation and discharged on the seventh postoperative day after a baseline chest radiograph, an ECG and a post operative echocardiography without any complication.

\section{Discussion:}

Congenital anomalous origin of the coronary arteries has been implicated in chest pain, sudden death, cardiomyopathy, syncope, dyspnea, ventricular fibrillation and myocardial infarction. ${ }^{9}$ In TOF, an anomalous origin of a major coronary artery across the RVOT is important surgically because a subpulmonic incision is usually made 
during a surgical repair. Usually 2\%-8\% of patients with TOF bear an anomalous LAD coursing either subepicardialy or remain buried in the myocardium across the RVOT. ${ }^{2,3}$. So identification of an anomalously arising LAD is an indispensable part of preoperative workup for patients with TOF to prevent accidental injury or transection inadvertently (resulting in serious myocardial ischaemia and death) during attempts to enlarge RVOT. ${ }^{2,3}$ The immediate and long-term outlook depends on the adequate and sustained relief of RVOTO without injury to the coronary artery supplying the significant portion of myocardium. The anomalous coronary crossing the RVOT presents a unique problem among the patients with TOF and hypoplastic pulmonary annulus because it cannot be enlarged with the standard technique using a trans-annular patch(TAP). Several techniques have been advocated for circumventing this situation for achieving total correction. ${ }^{8,10}$ Usually repair through the RA, RV and pulmonary artery(PA) is done but trans-atrial (TA) or a combination of TA and trans-pulmonary(TP) repair is possible only when the pulmonary annulus is considered adequate. ${ }^{10}$ Surgical treatment continues to evolve for annular hypoplasia associated with anomalous coronary vessel. ${ }^{8}$ When significant degrees of hypoplasia of pulmonary valve annulus and the MPA is frequently encountered, the most preferred surgical option in such a situation is placement of a conduit interposition between RV and MPA. RVOT reconstruction through different techniques like oblique ventriculotomy, tailored ventriculotomy, double barrel technique(two patch repair), pulmonary artery turn back technique and by using pulmonary homograft conduit. ${ }^{10}$ or even primary repair can be done without the use of a conduit by the translocation of MPA on the RVOT ${ }^{11}$ or transannular repair below a mobilized anomalous coronary artery. ${ }^{12}$

A hypoplastic pulmonary annulus with a normal coronary arterial pattern needs a trans-annular vertical incision followed by a patchplasty is the most preferred approach. $^{13}$ But the surgical technique in presence of anomalous LAD coronary artery across the RVOT will depend on the location and tortuosity of the anomalous coronary artery, the size of the MPA, annular diameter, the level and severity of RVOTO will determine it. ${ }^{3,8}$ If the PV annulus is normal/near normal size, obviating the need for TAP, the relief of RVOTO was accomplished through TA or combind TA-TP without any injury to the anomalous coronary artery. When the PV annulus is hypoplastic, necessitating a trans-annular patch. ${ }^{8}$ The close proximity $<10 \mathrm{~mm}$ of the LAD and the hypoplastic pulmonary valve ring preclude a TA-TP repair. ${ }^{14}$ The presence of an anomalous coronary artery across the site of intended reconstruction necessitates the use of MPA translocation or RVOT reconstruction under a mobilized LAD. $^{8}$ In our case, the MPA was shorter and narrower, annulus being hypoplastic with an anomalous LAD at a distance of about $1.2 \mathrm{~cm}$. from it across the RVOT. So it was not possible for us to do a communication between RV and MPA through translocation of MPA to RV. During extension of the pulmonary arteriotomy across the annulus to the infundibulum to repair the hypoplastic annulus, the sub-epicardially located LAD was deliberately sacrificed, so that annuloplasty as well as resection of RVOTobstructing fibromuscular bands could be done easily. After closure of VSD and resection of the infundibular septo-parietal fibromuscular bands, we did transpulmonary, trans-annular, and trans-ventricular pericardial patchplasty with the incorporation of monocusp pericardial valve after valvotomy.

Several surgical strategies have been used to protect the anomalously arising LAD. Bonchek in 1976 described the method of dissecting the coronary artery off the outflow tract with placement of an outflow patch beneath the artery. ${ }^{12}$ This technique carries the risk of stretching with resultant ischemia caused by right ventricular dilatation, which is not uncommon immediately after the operation. Although Tchervenkov et al. ${ }^{8}$ reported successful mobilization of the coronary arteries in children, this seems to be more securely used in adult patients due to the possibility of spasm of the coronary artery in children. In our case, there was no scope of mobilization of LAD, as it was not tortuous and elongated. So we deliberately sacrificed the LAD during infundibulotomy. Bypass grafting was the only method of restoring blood supply distal to the sacrificed LAD. Berry and McGoon were the first to report aorta-coronary bypass grafting with autologous saphenous vein in 1973. ${ }^{4}$ Shaffer, Berman, and Waldhausen reported another case of saphenous vein grafting for divided anomalous LAD in a 13-year-old boy. ${ }^{5}$ Although di Carlo and colleagues reported aortacoronary bypass with a polytetraflouroethylene (PTFE) graft in a 17-month-old boy, the graft was for a transected left coronary artery. ${ }^{15}$ Our case represents the use of a LIMA graft directly to the distal segment of sacrificed anomalous LAD. On the basis of various reports of use of the LIMA for coronary artery disease, we predict that the patient will do well. Extracardiac pulmonary conduit replacement may be an alternative method. However, the lack of availability and logistics of setting up of the homograft bank for the cryopreserved homograft's continue to encourage the non-conduit reconstructions 
of the right ventricular outflow tracts. ${ }^{16}$ Xenografts though offer off- the- shelf privileges, continue to be expensive. However, the probability of many re-operations for conduit replacement and the risk for ischemia due to compression of the coronary artery by the conduit are major disadvantages of this technique. ${ }^{3}$ Our technique offers the advantage of one final surgery for this patient by PA annuloplasty with the insertion of autologous pericardial patch containing a monocusp. It avoids the cost of conduits during the total corrections, thereby offering financial relief for the patients in our part of the world where public funding for cardiac surgery continues to be very low. Moreover, the short-term and the long-term results of the hetero-homografts are not much different. ${ }^{17}$ Schlichter et al have demonstrated in their 15 year followup study of fresh autologous pericardial conduits with an excellent short and long-term outcome and may not require any re-operations because of the size of the conduit(16 mm and more). ${ }^{18}$

A transverse ventriculotomy in the RV body below the LAD can be used for relief of infundibular stenosis and closure of the $\mathrm{VSD}^{19}$ or for a conduit to maintain RV-PA continuity but RV-tomy is dangerous and carries a risk for arrhythmia, RV dysfunction, low cardiac output syndrome(LCOS) and sudden death. ${ }^{20}$ We have avoided a ventriculotomy into the RV body, limited it to the outlet portion of RV ie., infundibulum which has a relatively minor functional contribution to RV stroke volume.

TA approach may be used, particularly when a ventriculotomy is dangerous due to unfavourable coronary anatomy, or when the RVOTO is discrete and low. The feasibility of TA repair is best judged preoperatively by calculating the Z-scores on angiocardiography. Accourding to traditional criteria, patients with pulmonary annulus Z-scores less than -2 are likely to require a transannular patch ${ }^{20}$ and a recent report, TA repair being successfully performed in patients with Z-scores of as low as $-4 .{ }^{20}$ Residual RVOT obstruction is an important factor that affects the outcome of corrective surgery for TOF. To avoid this, we have ensured that, in the flaccid arrested heart, a Hegar dilator at least two sizes bigger than that predicted by the Rowlatt chart ${ }^{20}$ for this particular patient could be passed easily.

The technique in addition to the TAP undoubtedly causes pulmonary failure. In our case, to reduce failure, a pericardial monocusp valve was implanted on a hypoplastic valve ring to treat TOF successfully, which offered our patient at least some short term competence of the pulmonary outflow tract. The balance between pulmonary stenosis (PS) and incompetency (PI) may be critical for preservation of ventricular function. A unified repair strategy that limit residual PS and PI is essential to maintain ventricular function. otherwise, over a prolonged period of time, the systolic pump function of RV will decrease at rest or progressively RV dilatation and failure which in turn predisposes to late life threating ventricular arrythmias and sudden death. Stenosis, in addition to insufficiency seemed to have been beneficial by promoting hypertrophy limiting RV dilation and enhancing myocardial contractility. Post-operatively, PI is well tolerated. Free PI causes RV dilatation that in turn compresses the LV.

In conclusion, there are several reconstructive techniques in TOF patients with the anomalous LAD artery crossing the RVOT with hypoplastic pulmonary annulus but is individualized depending on the age of patient, size of MPA, annular diameter, the level and severity of RVOTO, location and tortuosity of anomalous LAD and finally the expertness of the surgeon.

Follow up after 1 week and 1, 3 and 6 months: He is free of symptoms. Monocusp function is assessed by auscultation for presence of a split S2 and has no evidence of infarction or ischemia on the electrocardiogram ECG). Echocardiogram shows no dilatation of RV with competent monocusp and good left ventricular function having $65 \%$ LVEF, as well.

\section{References:}

1. Hekmat M, Foroughi M, Tehrani MMM,. Monfared MB et al. Tetralogy of fallot and associated coronary anomalies. Iranian Heart Journal 2004;5(1,2):39-42

2. Hurwitz RA, Smith W, King H, Girod DA, Caldwell RL. Tetralogy of Fallot with abnormal coronary artery: 1967 to 1977. J Thorac Cardiovasc Surg 1980; 80: 129-34.

3. Humes RA, Driscoll DJ, Danielson GK, Puga FJ. Tetralogy of Fallot with anomalous origin of left anterior descending coronary artery. Surgical options. J Thorac Cardiovasc Surg 1987; 94: 794-7

4. Berry BE, McGoon DC. Total correction for tetralogy of Fallot with anomalous coronary artery Surgery 1973;74: 894-8

5. Shaffer CW, Berman W, Waldhausen JA. Repair of divided anomalous anterior descending coronary artery in tetralogy of Fallot. Ann Thorac Surg 1979; 27: 250

6. O'Sullivan J, Bain H, Hunter S, Wren C. End-on aortogram: improved identification of important coronary artery anomalies in tetralogy of Fallot. Br Heart J 1994;.71 :102-6.

7. Kim SY. Seo JB, Do DH, Heo JN, Lee JS, Song JW et al.:Classification and MDCT. Radiographics 2006;26:317-34 
8. Tchervenkov CI, Pelletier MP, Shum-Tim D, Beland MJ, Rohlicek C. Primary repair minimizing the use of conduits in neonates and infants with tetralogy or double-outlet right ventricle and anomalous coronary arteries. J Thorac Cardiovasc Surg 2000;119: 314-23.

9. Angelini P, Velasco JA, Flamm S. Coronary anomalies: incidence, pathophysiology and clinical relevance. Ciirculation. 2002;105: 2449-54.

10. Brizard CP, Mas C, Sohn YS, Cochrane AD, Karl TR. Trans atrial- Transpulmonary Tetralogy of Fallot repair is effective in the presence of anomalous coronary arteries. J Thorac Cardiovascular Surg 1998; 119: 314-23

11. Lecompte Y,Neveux JY, Leca F, et al. Reconstruction of the pulmonary outflow tract without prosthetic conduit.J Thorac Cardiovasc Surg 1982;84:727-33

12. Bonchek LI. A method of outflow tract reconstruction in tetralogy of Fallot with anomalous anterior descending coronary artery. Ann Thorac Surg 1976; 21:451-3.

13. Hallman GL, Cooley DA. Surgical treatment of tetralogy of Fallot: experience with indirect techniques. J Thorac Cardiovasc Surg 1963; 46:419-23
14. O’Blenes SB, Freedman RM, Coles JG. Tetralogy of Fallot with anomalous LAD: Repair without conduit. Ann Thorac Surg 1996; 62:1186-88

15. Di Carlo D, De Nardo D, Ballerini L, Marcelletti C. Injury to the left coronary artery during repair of Tetralogy of Fallot: successful aorta-coronary polytetrafluoroethylene graft. J Thorac Cardiovasc Surg 1987;93: 468

16. Kumar RK, Srivastava S. Pediatric Heart Care in India, In press, Heart

17. Powell AJ, Lock JE, Keane JF, Perry SB. Prolongation of conduit life span by percutaneous stent implantation. Intermediate results. Circulation 1995; 92:3282-8.

18. Schichter AJ, Kreutzer C, Mayorquim RC, Simon JL, Roman MI, Vasquez H, et al. Five to fifteen year follow-up of fresh autologus pericardial valved conduits. J Thorac Cardiovasc Surg 2000;119:869-79.

19. Meyer J, Chiariello L, Hallman GL, et al. Coronary artery anomalies in patients with Tetralogy of Fallot. J Thorac Cardiovasc Surg 19

20. Airan B, Chaudhary SK,Kumar HVJ et al . Total transatrial correction of TOF: No outflow patch technic . Ann Thorac Surg 2006;82: 1316-21 